\title{
Wo bist du, Sonne, geblieben!
}

\author{
Von der Ironie einer Geschichte, die nicht sterbenslang- \\ weilig ist
}

Ralph Kunz

\section{Fin de siècle}

In seinem letzten Roman Der Stechlin kreist Theodor Fontane (1819-1898) um die Figur des melancholischen Gutsbesitzers Dubslav von Stechlin, der weltfremd lebt, aber weltweise fühlt - und stirbt kurz darauf. Das Bewusstsein der verknappten Lebenszeit schärft den Blick für das Unfertige - auch in der eigenen Biographie - und nährt die Sehnsucht nach einer Schau des Ganzen. Es wächst das Bedürfnis, durch Bilanzierung etwas abzuschliessen, das nicht rund gelaufen ist, und andern seine Geschichte zu erzählen, wenn die Tage gezählt sind. Spürte Fontane, dass sein Ende naht? Schuf er sich mit dem Stechlin ein Vermächtnis?

Ich finde ja. Theodor Fontanes Roman erzählt vom Sterben - so traurig und doch so schön, so frech und doch so fromm, so ironisch und doch so ergreifend. Fontane erzählt doppelbödig und zwiespältig - in einzigartiger Geschlossenheit, wie ein Mann »im besten Alter« mit seinem Sterben (nicht) fertig wird. Sozusagen eine thanatologische Lektüre par excellence. Man kann den Roman auch gerontologisch lesen. ${ }^{1}$ Schliesslich wird Stechlin älter, bevor er stirbt. Ich meine, man muss das Buch von hinten lesen - vom Schluss her und dann wieder von vorne beginnen. Ist das nicht der Sinn der Sterbenserzählung? Dass sie denen, die noch nicht gestorben sind, helfen, mit dem Leben etwas anzufangen und mit dem Sterben (doch noch) fertig zu werden. Darum lohnt es sich, sich mit Theodor Fontanes Stechlin eingehender zu beschäftigen.

Wie jedes gute Buch lädt auch dieses seine Leserinnen und Leser ein, selber nach Antworten zu suchen - in langen Wanderungen rund um den See, das Dorf, das Schloss und die Person, die allesamt

\footnotetext{
1 Vgl. meinen Essay Das Glück im Unglück der späten Jahre. Altern als Vexierbild, in: Familiendynamik 40 (2015/4) 268-275. Der vorliegende Text ist eine überarbeitete und erweiterte Fassung der dort angestellten Überlegungen.
} 
Stechlin heissen. Der alte Major ausser Dienst hat es mir angetan, weil er ein gläubiger Skeptiker ist. Man muss den Stechlin mögen. Sonst wird es sterbenslangweilig. Der Roman ist nicht gerade ereignisreich. Man könnte den Inhalt in Anlehnung an einen Kinohit so auf den Punkt bringen: $A$ Wedding and a Funeral. Fontane selbst beschrieb die Handlung ironisch ganz ähnlich: "Zum Schluß stirbt ein Alter und zwei Junge heiraten sich; - das ist so ziemlich alles, was auf 500 Seiten geschieht. $\ll^{2}$ Das ist natürlich nicht wahr. Es geschieht unendlich viel mehr - aber es geschieht im Verborgenen.

Dass der Roman Der Stechlin heisst, ist denn auch absichtlich mehrdeutig. Ist der See gemeint? Mit dem See hat es nämlich seine Bewandtnis. "Alles still hier. Und doch, von Zeit zu Zeit wird es an ebendieser Stelle lebendig." Wenn draussen in der Welt grosse Dinge geschehen, »dann regt's sich auch hier (Fontane 2014, 665). ${ }^{3}$ Das wissen alle, die den Stechlin umwohnen, auch wenn sie es nie mit eigenen Augen gesehen haben. Der See bleibt meistens still und birgt sein Geheimnis. Es ist ein Gleichnis für die Glaubensthematik, die subtil das Werk durchzieht. Die geheimnisvolle Verbindung zwischen der grossen und der kleinen Welt - verpackt in den Mythos - wird geglaubt, aber nicht geschaut. Niemand hat das Wunder je gesehen.

Fontane schreibt seinen letzten Roman 1897. Es ist ein »Fin de siècle«-Roman der konservativeren Art. Drei Jahre vor dem Anbruch des neuen bilanziert Fontane das alte Jahrhundert - lässt es Revue passieren in den Memoiren des alten Majors, der sich mit allen möglichen Leuten unterhält. Es wird viel geplaudert. Über den alten König, die neuen Ideen der Moderne, die alles fortzuschwemmen scheint. Stechlin ist ein Konservativer, was die Politik angeht, und ein Agnostiker in Glaubensdingen, aber er lässt andere zu Wort kommen, und am Ende verschlägt es ihn dann doch nicht in die Politik. In seinem heiteren abgeklärten Wesen, das zugleich eine tiefe religiöse wie politische Zerrissenheit offenbart, spiegelt sich der Autor:Theodor Fontane schafft sich mit dem Stechlin sein alter ego, das mit dem alten Jahrhundert stirbt - im Bewusstsein, dass mit ihm eine Epoche zu Ende geht. Auch eine "Zeit» kann sterben. Der alte Stechlin alias Fontane hat denn auch seine liebe Mühe mit dem "Fortschritt". Das alles ist ihm zutiefst suspekt, und er erkennt, dass er das Neue nicht mehr versteht. Ein Jahr nach derVeröffentlichung stirbt der Autor.

2 Zitiert nach Helmuth Nürnberger, Theodor Fontane, Reinbek 1998, 157.

3 Ich zitiere aus den Gesammelten Werken: Theodor Fontane, Gesammelte Werke, Köln 2014 (Original: Theodor Fontane, Der Stechlin, Berlin 1899). 
Peter Härtling, mit dem ich die Liebe zum Stechlin teile, schreibt:

»Fontane hat sich die Geschichte einer gewünschten Existenz geschrieben. Er ist mit Dubslav von Stechlin so verbunden wie der Stechlin-See mit der fernen Welt. Je tiefer man in das Buch gerät, um so mehr glaubt man, einem Selbstgespräch zuzuhören, einem unendlichen, Figuren sammelnden, Figuren verlierenden Monolog, der sich immer wieder in Dialogen verzweigt, im Grunde Dialogen für eine Stimme. ${ }^{4}$

\section{Es lebe der Stechlin!}

Fontane zeichnet also sein Bild eines liebenswürdigen Herrn. "[S]chon ein gut Stück über Sechzig hinaus, war [er] der Typ eines Märkischen von Adel, aber von der milderen Observanz, eines jener erquicklichen Originale, bei denen sich selbst die Schwächen in Vorzüge wandeln." (667) Er ist ein heiterer Skeptiker, der die Selbstironie pflegte, "weil er seinem ganzen Wesen nach überhaupt hinter alles ein Fragezeichen machte« (ebd.). Seine tiefe aus dem Herzen kommende Humanität lässt ihn allergisch werden gegen Überheblichkeit. »Er hörte gern eine freie Meinung, je drastischer und extremer, desto besser. Dass sich diese Meinung mit der eigenen deckte, lag ihm fern zu wünschen. «Paradoxien waren seine Passion, und sein Credo lautet: ॥Unanfechtbare Wahrheiten gibt es überhaupt nicht, und wenn es welche gibt, so sind sie langweilig." (Ebd.)

Stechlin erlebte glückliche Tage, aber sie waren von kurzer Dauer. Seine Frau starb früh. Dreissig Jahr lebte er allein als Witwer. Sich wieder mit einer neuen Frau zu verbinden, widerstand ihm, »halb aus Ordnungssinn und halb aus ästhetischer Rücksicht" (668). Die Begründung spricht für sich - und lässt durch den Kommentar des Erzählers auch die Glaubensskepsis Fontanes durchschimmern: ")Wir glauben doch alle mehr oder weniger an eine Auferstehung (das heisst, er persönlich glaubte eigentlich nicht daran), sund wenn ich dann oben ankomme mit einer rechts und einer links, so is das doch immer eine genierliche Sache.« (Ebd.)

Das beschauliche, wenn auch einsame Leben des Majors nimmt eine dramatische Wende, als sich eine Krankheit einstellt - ein Leiden, das dem Alten die Lebenskraft raubt. Langsam wird ihm klar, dass es

\footnotetext{
4 Peter Härtling, Theodor Fontane: Der Stechlin, in: DIE ZEIT, Nr. 11, 09. März 1979, 51, aus dem Archiv bei ZEIT ONLINE: http://www.zeit.de/1979/11/derstechlin (10.11.2016).
} 
mit ihm zu Ende geht. Er ahnte es freilich schon vorher. Immer wieder taucht das Thema des Sterbens in den Plaudereien auf.Wenn er sich den Freunden seines Sohnes als "vergrätzten alten Einsiedler" (675) vorstellt oder im Gespräch mit dem Sohn auf die Fantasie des ewigen Fortlebens zu sprechen kommt und meint, ein egoistisches Gefühl bestehe darauf, »dass die Stechline ewig weiterleben, wenn es sein kann in aeternum. Ewig weiterleben; ich räume ein, es hat ein bisschen was Komisches, aber es gibt wenig ernste Sachen, die nicht auch eine komische Seite hätten ...« (704), so spricht hier der müde Autor, der seinen letzten Roman schreibt und hin und her macht zwischen dem Ernst und der Komik der Situation. Ob er sich mit dem Stechlin ein Denkmal setzt? Es bleibt schillernd.

Das gilt auch für die Figur des guten Pastor Lorenzen: ein neumodischer Theologe, der sich für den Sozialismus à la Bebel erwärmen kann und mit dem sich der Major gut unterhält. Der Alte schätzt ihn - weil »er nicht von Erlösung und Unsterblichkeit spricht» (986). Lorenzen steht für einen offenen Protestantismus ein - ganz anders als Stechlins Schwester Adelheid, die im Kloster Wutz lebt. Sie führt das dortige Damenstift, ist erzkonservativ und missbilligt die liberalen Anschauungen ihres Bruders. Mit ihr hat der alte Major seine Müh' und Not. Kratzbürste und Misanthropin, die sie ist, müsste man dem Mann, wenn sie einer jemals heiraten wollte, das eiserne Kreuz für seine Tapferkeit aushändigen. Meint der Bruder.

Die Gegenfigur zur Schwester aus Wutz ist die Beinah-Schwiegertochter Melusine bzw. die Schwester von Armgard, die Waldemar - der junge Stechlin - heiraten wird. Fontane-Exegeten erkennen eine wiederkehrende Frauenfigur. Fontane und seine Frauen! - das ist ein weites Feld. Darum nur so viel: Wenn der Alte eine Passion hat furr das Paradoxe, ist sie die ausgleichende Mitte, weniger schillernd als dieser und doch Schiller nahe. Denn sie ist die schöne Seele. Sie ist meerjungfräulich, so klar wie der Stechlin-See und doch Geheimnisse bergend. Sie heiratet nicht und ist vielleicht darum so heiter, abgeklärt und weise. Fontane legt ihr den Spruch in den Mund: "Alles Alte, soweit es Anspruch darauf hat, sollen wir lieben, aber für das Neue sollen wir recht eigentlich leben." (845)

Schöner kann man den Gegensatz nicht versöhnen. Wer, wenn nicht der alte Fontane, spricht hier? Er hat mehr als nur zwei Seelen in seiner Brust und offensichtlich auch eine schöne. Melusine hat auch das Schlusswort. Sie schreibt nach der Beerdigung des Stechlin - ihn unbewusst zitierend - an ihren Seelenverwandten Pastor Lorenzen: 
"Und nun, lieber Pastor, noch einmal das eine. Morgen früh zieht das junge Paar in das alte Herrenhaus ein, meine Schwester und mein Schwager [...], es ist nicht nötig, dass die Stechline weiterleben, aber es lebe der Stechlin.«(1008)

\section{Wo bist Du, Sonne, blieben?}

Es ist denn auch der Schluss dieses Romans, der eine Intensität hat, die unter die Haut geht. Als der Alte realisiert, dass er ernsthaft erkrankt ist, ruft er zuerst nach den Ärzten. Das macht er widerwillig, weil er nicht viel von ihnen hält. Er sollte Recht behalten. Die ärztliche Kunst ist am Ende. "Nun also bleibt noch der liebe Gott." (985) Oder doch nicht? Jedenfalls lässt Stechlin eine der Buschen - eine Kräuterhexe - holen. Sie macht ihm einen Trank: grüne Tropfen und Katzenpfötchentee. Das bringt zunächst tatsächlich etwas Linderung, aber die Krankheit ist stärker. Nun hat aber das Gerücht, dass sich der Major auf die Pfade des Aberglaubens begibt, die Aufmerksamkeit der frommen Seelen gefunden. Und die setzen zum Angriff an. Ihre Stunde ist gekommen. Fontane - Meister des Doppelbödigen - schreibt:

"Es schlug zwölf, als Dubslav vom Portal her wieder den Flur passierte. Dabei sah er nach dem Hippenmann hinauf und zählte die Schläge. 'Zwölf‘, sagte er, sund um zwölf ist alles aus und dann fängt der neue Tag an. Es gibt freilich zwei Zwölfen, und die Zwölf, die da oben jetzt schlägt, das is die Mittagszwölf.Aber Mittag! [...] Wo bist du, Sonne, geblieben!« (951)

Doppelbödiger geht's nicht. Stechlin deutet die Zeichen. Er hört den Mitternachtsruf, obwohl es heller Mittag ist und zitiert ein Abendlied. In der zweiten Strophe von Gerhardts Nun ruhen alle Wälder heisst es: "Wo bist du, Sonne, blieben? / Die Nacht hat dich vertrieben, / die Nacht, des Tages Feind. / Fahr hin, ein andre Sonne, / mein Jesus, meine Wonne, / gar hell in meinem Herzen scheint. $\aleph^{5}$ Was hört der Alte? Mittag oder Mitternacht? Was sieht der Alte? Die Sonne, die untergeht, oder die Herzenssonne? Es kippt hin und her. Nachdem es also zwölf geschlagen hat, will Stechlin Zeitung lesen, aber er bleibt unruhig. Er ahnt, es kommt Besuch. Ob es wohl der Pastor Koselger mit seinen Heilsgütern ist? Der Alte sinniert:

\footnotetext{
5 Zitiert aus dem Evangelisch-reformierten Gesangbuch, Zürich 1998, 594.
} 
"Ja, die Heilsgüter, die sind ganz gut. Versteht sich. Ich werde mich nicht so versündigen. Die Kirche kann was, is was, und der alte Luther, nu der war schon ganz gewiss was, weil er ehrlich war und für seine Sache sterben wollte. Nahe dran war er. Eigentlich kommt's doch immer bloss darauf an, dass einer sagt, >dafür sterb ich gleich.« (952)

Solcherart sein Credo bedenkend, bekommt Dubslav Stechlin tatsächlich Besuch. Die Prinzessin und fromme Frau des Oberförsters ist gekommen, um die Seele des Majors zu retten (953). Es entspannt sich ein Glaubensgespräch, in dessen Verlauf der alte Major bekennt, dass er es mit der Seele genauso wie dem Körper halte.

"Wenn sich meine arme Seele ängstigt, dann nehm ich mir Trost und Hilfe, so gut ich sie gerade finden kann. [...] Alle Worte, die von Herzen kommen, sind gute Worte, und wenn sie mir helfen $[\ldots]$, so frag ich nicht viel, ob es sogenannte richtige Worte sind oder nicht." (954)

Stechlin fasst in einen Satz, was hundert Jahre später die religionssoziologischen Auguren mit "Körperindex", "Deinstitutionalisierung" und "Bastelreligiosität» erklären: Er hat eine nach pragmatischen Gesichtspunkten selbst bestimmte reflexive Spiritualität, die sich von korrekter Religiosität distanziert. Die dogmatisch korrekte Prinzessin kann diesem Bekenntnis natürlich wenig abgewinnen. "Ihr bis dahin verbindliches Lächeln", beschreibt Fontane den Atmosphärenwechsel, "war sichtlich in raschem Hinschwinden." (Ebd.)

\section{Lassen wir die heikle Frage}

Ein zweites Seelsorgegespräch mit Pastor Lorenzen verläuft harmonischer, obwohl es mit einem Vorwurf des Alten beginnt, der beinahe schulbuchmässig ist. Fontane lässt Stechlin sagen: „Warum kommen Sie erst jetzt? Und das heisst dann Seelsorge!« Der Pastor antwortet charmant:

"Herr von Stechlin, Ihre Seele macht mir, trotz dieser meiner Vernachlässigung, keine Sorge, denn sie zählt zu denen, die jeder Spezialempfehlung entbehren können. Lassen Sie mich sehr menschlich, ja für einen Pfarrer beinah lästerlich sprechen. Aber ich muss es. Ich lebe nämlich der Überzeugung, der liebe Gott, 
wenn es mal so weit ist, freut sich, Sie wiederzusehen. Ich sage, wenn es so weit ist. Aber es ist noch nicht so weit.« (964)

Dass sich Lorenzen nun doch auf eine Art Unsterblichkeit einlässt - wenn auch in scherzhafter Weise -, ist insofern bemerkenswert, als der Pastor tatsächlich glaubt, es sei noch nicht so weit. Er sollte sich täuschen. Wie auch immer: Nach dieser Steilvorlage des Seelsorgers darf man auf ein Glaubensgespräch hoffen. Aber Stechlin weicht (scheinbar) aus. Er sagt: "Lassen wir die heikle Frage.« Und dann schwadroniert er über das Zeitungslesen und meint: "Beiläufig natürlich nur vom alleregoistischsten Zeitungsleserstandpunkt aus - ein wahres Glück, dass es Unglücksfälle gibt, sonst hätte man von der Zeitungslektüre so gut wie gar nichts. "(Ebd.)

Stechlin lobt auch das Heldische. "Je schlechter es einem geht, je schöner kommt einem so was kavalleristisch Frisches und Übermütiges vor.« (Ebd.) Das Gespräch nimmt eine neue Wende, als Stechlin auf einen Helden anderen Kalibers zu sprechen kommt: einen Nordpolfahrer namens Greenley, der sich entschlossen hatte, einen Kameraden, der Lebensmittel stahl, hinterrücks zu erschiessen. Lorenzen meint: Dieser Mut imponiere ihm. Dass einer Schmach und Schimpf auf sich lade, um andere Kameraden zu retten. Und so mündet das Gespräch über das Seelenheil in ein kreuzestheologisch gedeutetes Musterbeispiel für Verantwortungsethik und einer sublimen Kritik an der Gesinnungsethik eines Übermenschen. ${ }^{6}$

Bemerkenswert ist ein drittes Gespräch, in dem die knorrige Adelheid sich zu Wort meldet. Sie wäscht ihrem kleinen Bruder so richtig die Kappe. Hören wir die innere Stimme des alternden ExPietisten Fontane?

»Es soll jetzt viele solche geben, denen ihr Humor und ihre Rechthaberei viel wichtiger ist als Gläubigkeit und Apostolikum. Denn sie sind sich selber ihr Glaubensbekenntnis. Aber, glaube mir, dahinter steckt der Versucher, und wohin der am Ende führt, das weisst du, - so viel wird dir ja wohl noch geblieben sein. ıIch hoffe es`, sagte Dubslav.« (965)

Hofft er es?

${ }_{6}$ Fontane (967) nimmt hier kritisch Bezug auf Nietzsches Begriff des Übermenschen, den dieser zuerst in Also sprach Zarathustra (1883-85) entfaltet. "Jetzt hat man statt des wirklichen Menschen den sogenannten Übermenschen etabliert; eigentlich gibt es aber bloß noch Untermenschen, und mitunter sind es gerade die, die man durchaus zu einem ,Über machen will. Ich habe von solchen Leuten gelesen und auch welche gesehn. Ein Glück, daß es, nach meiner Wahrnehmung, immer entschieden komische Figuren sind, sonst könnte man verzweifeln." 


\section{Die engelhafte Agnes}

Alle Figuren, mit denen der alte Major sich bespricht, vermögen nicht zu bewirken, was schliesslich nur das Kind vermag. Peter Härtling erzählt in seinem kurzen Artikel in der ZEIT, wie er den Stechlin als 20-, 30-,40- und 50-Jähriger gelesen und immer wieder Neues entdeckt habe. Spannend finde ich, wie der alte Leser den Schluss neu liest:

"Jetzt, mit 74, wage ich es, lese ich von seinem Sterben, wiederholt Tränen zu vergießen, ihm mit der kleinen Agnes, der Enkelin der von den Dorfbewohnern gemiedenen Kräuterhexe, zum Abschied einen Strauß Schneeglöckchen zu bringen und mir als Nachruf den Satz zu wünschen, mit dem der Pastor die Grabrede schließt: /Er war das Beste, was wir sein können, ein Mann und ein Kind.«

Was den Stechlin tröstet und seinen treuen Leser rührt, ist die beste Seelsorge, die der Sterbende empfängt. Was in den Gesprächen mit seinen Besuchern unter den Tisch gefallen ist, kommt jetzt zum Vorschein. Die Empfänglichkeit des Alten für das Reine, Kindliche und Schöne. Es gab schon vorher Hinweise darauf: etwa, wenn Fontane dem leidenden Stechlin helle Momente schenkt, in denen

»ein stilles Behagen über ihn gekommen [war], und er überdachte, wie manche Freude das Leben doch immer noch habe.Vor ihm, in den Parkbäumen, schlugen die Vögel, und ein Buchfink kam bis auf den Tisch und sah ihn an, ganz ohne Scheu. Das tat ihm ungemein wohl. Etwas ganz besonders Schönes im Leben ist doch das Vertrauen, und wenn's auch bloß ein Piepvogel is, der's einem entgegenbringt. Einige haben eine schwarze Milz und sagen: alles sei von Anfang an auf Mord und Totschlag gestellt. Ich kann es aber nicht finden.« (963)

Das Vögelchen ist wie der Zwölferschlag ein Zeichen - mehrdeutig, aber nicht zwiespältig. Auch das Mädchen, die Enkelin der Kräuterhexe, die den Sterbenden tröstet, ist ein solches Vögelchen. Stechlin möchte, dass sie bei ihm bleibt. »Ich freue mich, wenn ich das Kind sehe." (978) Und sie kommt - um ihn zu unterhalten wie der Piepvogel. Der Alte sorgt sich, dass dem Kind die Unterhaltung fehle. Aber dann hilft das alte Ortsmuseum. "Es waren glückliche Tage für Agnes. Aber fast noch glücklichere für den Alten." (980) Ja, der alte Dubslav freute sich des Kindes. Aber so wohltuend ihm seine Gegenwart war, 
"so war es auf die Dauer doch nicht viel andres, als ob ein Goldlack am Fenster gestanden oder ein Zeisig gezwitschert hätte. Sein Auge richtete sich gerne darauf, als aber eine Woche und dann eine zweite vorüber war, wurd' ihm eine gewisse Verarmung fühlbar, und das so stark, daß er fast mit Sehnsucht an die Tage zurückdachte, wo Schwester Adelheid sich ihm bedrücklich gemacht hatte." (Ebd.)

Stechlins Sehnsucht nach Menschen, ja selbst nach Auseinandersetzungen, ist Ausdruck eines inneren Kampfes - er will nicht gehen, er will leben. Aber immer deutlicher steht es vor Augen, dass gegen den Tod kein Kraut gewachsen ist. Auch der bittere Katzenpfötchentee hilft nicht. Die Stunde naht. Der Alte fragt das Kind:

"'Agnes, gefällt es dir hier? ’ Ja, gnäd'ger Herr, es gefällt mir hier.` `Und ist dir auch nicht zu still? > Nein, gnäd'ger Herr, es ist mir auch nicht $\mathrm{zu}$ still. Ich möchte immer hier sein.< >Na, du sollst auch bleiben, Agnes, so lang es geht. Und nachher. Ja, nachher ... Das Kind kniete vor ihm nieder und küßte ihm die Hände." (Ebd.)

Dubslav Zustand verschlechtert sich. Er fühlt, daß es zu Ende geht und spricht sein letztes Wort - ein einsames Bekenntnis auf dem Sterbebett:

" Das $>$ Ich $<$ ist nichts, - damit muß man sich durchdringen. Ein ewig Gesetzliches vollzieht sich, weiter nichts, und dieserVollzug, auch wenn er 'Tod heißt, darf uns nicht schrecken. In das Gesetzliche sich ruhig schicken, das macht den sittlichen Menschen und hebt ihn. Er hing dem noch so nach und freute sich, alle Furcht überwunden zu haben. Aber dann kamen doch wieder Anfälle von Angst, und er seufzte: \Das Leben ist kurz, aber die Stunde ist lang.« (993)

Es war eine schlimme Nacht. Aber nach Golgatha beginnt der nächste Morgen mit einem Bild der Auferstehung - oder eine Salbung - auf jeden Fall eine Tat der Liebe:

"Die Kleine trat auch leise durch die Balkontür auf die Veranda hinaus und ging auf das Rundell zu, um nach ein paar Blumen zu suchen. Sie fand auch allerlei; das beste waren Schneeglöckchen. Und nun ging sie, mit den Blumen in der Hand, noch ein paar mal auf und $\mathrm{ab}$ und sah, wie die Sonne drüben aufstieg. Sie fröstelte. Zugleich aber kam ihr ein Gefühl des Lebens. Dann trat sie wieder in das Zimmer und ging auf den Stuhl zu, wo 
Dubslav saß. Engelke, die Hände gefaltet, stand neben seinem Herrn. Das Kind trat heran und legte die Blumen dem Alten auf den Schoß. `Dat sinn de ihrsten`, sagte Engelke, sun wihren ook woll de besten sinn.«(994)

\section{Das Vermächtnis}

Es gibt so viele Lebens- und Sterbensgeschichten wie es Menschen gibt, und jeder Versuch, diese Vielfalt auf ein Metanarrativ zu reduzieren, führt zu einerVerarmung. Darum brauchen wir die Literatur. Sie bewahrt die Fülle der Erzählungen von Menschen, deren Tage gezählt sind. Fontanes melancholisches Spätwerk ist einzigartig. Natürlich gibt es Lesarten, die das Werk durchsichtig machen für das typisch Menschliche. Was erzählt wird, bewegt, weil es einen Resonanzboden findet im eigenen Erleben. Es ist nicht das Allgemeine, das berührt, sondern das Ureigene im Ähnlichen. Ich lasse mich vom Roman zum Gespräch über mein Sterben verführen, weil es so gar nicht sterbenselend ist.

Erik Erikson spricht vom Vermächtnis als dem Wunsch, etwas von seinem Werk an die nächste Generation weiterzugeben: Das kann ein geistiges Erbe sein. Es ist das Bedürfnis, eine Spur der Erinnerung zu hinterlassen und seine Geschichte zu erzählen. Die letzte Entwicklungsaufgabe ist vom Paradox geprägt, dass diejenigen, die sie annehmen, nicht wissen, ob sie ihnen gelingt. Ist das nicht ein fantastischer Grund, ein Buch zu schreiben und seinen Abgang vorweg zu nehmen? Was hat man davon, wenn man anderen etwas von sich hinterlässt? Lebt man dann weiter? Ist das - mit den Worten des alten Majors - nicht doch egoistisch? Letztlich müssen es die anderen sagen. Es ist ja auch in diesem wunderbaren Fontane-Roman so, dass am Schluss - am Grab - der Pastor Lorenzen für den Stechlin das Bekenntnis sprach und sich zu ihm bekennt, wenn er sagt, er "war recht eigentlich das, was wir überhaupt einen Christen nennen sollten. Denn er hatte die Liebe. [...] Er ist nun eingegangen in seines Vaters Wohnungen und wird da die Himmelsruhe haben, die der Segen aller Segen ist.« (998)

Am Ende kommt Stechlin sehr gut weg. Pastor Lorenzen schließt die Grabrede mit dem Satz: »Er war das Beste, was wir sein können, ein Mann und ein Kind." Was in der Stunde des Todes noch unentschieden war, löst sich in der Rede am Grab in Minne auf. Mit Blick auf die Phasen der Unsicherheit, des Zweifels und der Unruhe, ist das wenig tröstlich - im Nachhinein ist alles gut.Vor dem Finale fragt man sich: Muss man, um in den Genuss dieser segensvollen 
Ruhe zu kommen, nicht doch ein frommer Christ sein? Fontane hat mit dieser Frage gerungen und - vor dem Finale - für sich einen versöhnlichen Schluss gefunden.

Letztlich geht es auch in dieser wie in jeder Erzählung um die Annahme der Endlichkeit. Dazu braucht es nicht dringend einen lieben Gott. Es ist, wie Stechlin tapfer bekennt, ein »sich ruhig schicken in das Gesetzliche, das macht den sittlichen Menschen und hebt ihn" (993). Aber Fontane zeichnet gerade keinen Übermenschen, der heroisch abtritt. Das macht den Stechlin so menschlich. Dass er sich dieser Ambivalenzerfahrung stellt.

»Unsere Tage zu zählen, das lehre uns, damit wir ein weises Herz gewinnen! Lehre uns bedenken, daß wir sterben müssen, auf daß wir klug werden", sagt der Psalmist (Ps 91:12). Wir sind in Geschichten verstrickt, die meistens nicht aufgehen, und am Ende geht es auch nicht auf, warum wir klug werden sollen, wenn wir sowieso sterben müssen. Es sei denn, das Erzählen selbst wird zum Akt, der die Endlichkeit menschlich macht, weil er diejenigen, deren Leben zu Ende geht, mit denen verbindet, deren Leben weitergeht. Das Ambivalenzkonzept sensibilisiert für die Spannung, die sich im endlichen Leben aufbaut und die sich lösen kann, wenn einer erzählt, was ihn - weil es mit ihm zu Ende geht - quält und plagt. Das schliesst die Bereitschaft mit ein, den Traum der Perfektion aufzugeben, sich mit dem Unfertigen zu begnügen und sich die Geschichte vom Ende immer wieder neu erzählen zu lassen.

\section{Das Bild des himmlischen Kindes}

Fontanes Stechlin muss sterben. Er weiss es. Nur das bleibt ihm noch: sich darüber zu freuen, dass es weiter geht - nicht mit den Stechlinen, aber mit diesem Kind, der Agnes, die Enkelin der Buschen. Das ist berührend, weil es ein Glück ist, das nur im Unglück des Hingangs erlebt werden kann. Im Sterben zeigt sich ein neues Leben. Für den Stechlin geht es durch den Dornenwald, aber am Ende kommt ein Kind zur Welt. Es macht auch dieser Schmerz des Sterbens den Anschein eines produktiven Schmerzes. Als ob es am Ende um eine Neugeburt ginge. So verbinden sich, wenn auch ganz fein und leise, Symbole der biblischen Botschaft mit dem Bild natürlicher Hoffnung. Es ist dieses Bild, das Härtling zu Tränen rührt. Die echte Zuneigung der 10-Jährigen, die dem Alten Blumen in den Schoss legt und am Grab wie ein Schlosshund schluchzt und nicht mehr leben will. 
Fontane ist ein Meister. Es wird nicht einmal hier kitschig. Das Bild schillert. Es kippt, wenn man weiss oder ahnt, was der Alte ahnte, als er das Mädchen im Abendlicht der untergehenden Sonne zum ersten Mal sah. Er sass damals am See, da begegnet ihm Mutter und Kind. Mit der Mutter, erfährt der Leser nur beiläufig, hatte Dubslav ein kurzes Verhältnis. Dass er die Agnes gezeugt hat, dass sie seine uneheliche Tochter ist, schwante auch ihm erst in diesem Augenblick! Agnes ist also in mehrfacher Hinsicht die Botin des Lebens: Sie ist Symbol des Reinen und die Enkelin der Buschen, die Blumen sammelt und eine Verbindung herstellt zur alten Religion. Sie ist auch sein Kind. Das alles tröstet den Sterbenden. Agnes ist einerseits das engelsgleiche Kind, dem es am Grab das Herz zerreisst, weil sie ihren Stechlin vermisst, und sie ist andererseits das uneheliche "Lütt", das ihren Vater beweint. Das ist zwiespältig, aber vielleicht - mit einer differenzierten Ambivalenz gesagt - sind es Bilder, die nebeneinander stehen und ineinander übergehen, ohne einander zu zerstören. Das ist schön, aber es ist nicht die letzte glückselige Schau. Die Differenz offen zu lassen, ist die Aufgabe der biblischen genauso wie der literarischen Bilder. Sie geben Unsichtbares zu erkennen und lassen jetzt schon auf das Letzte hoffen, aber (noch) nicht schauen.

\section{Und ist doch rund und schön}

Stechlin fragt: Wo ist die Sonne blieben? Und zitiert das fromme Abendlied, das in der Aufklärungszeit eine heisse Debatte auslöste, weil es heisst: Nun ruhen alle Wälder. Die Neunmalklugen, die keinen Widerspruch duldeten, weigerten sich, diesen Nonsens zu singen. Jetzt, da man wisse, dass auf der anderen Seite der Erde die Gegenfüssler just in der Stunde wachen, in der auf dieser geschlafen wird. Mitternacht ist auch Mittag. Darum singe man besser: Es ruht ein Teil der Wälder. Matthias Claudius - ein Zeitgenosse dieser Kritiker - schrieb als Antwort auf diese Debatte ein wunderbares Gegenlied. Nicht die Sonne, sondern der angeleuchtete Mond ist der Himmelskörper, der in der Nacht Licht gibt. Die Dämmerung, der dunkle Wald ja selbst der Nebel werden zur tröstlichen Verhüllung - und am Himmel steigt ein Zeichen auf. Es ist der Halbmond. Er ist nur halb zu sehen und ist doch rund und schön. Wie der Roman von einem, der stirbt. Es lebe der Stechlin!

- Ralph Kunz ist Professor für Praktische Theologie an der Theologischen Fakultät Zürich und Mitglied der Leitung des Zentrums für Gerontologie der Universität Zürich. Seine Forschungsschwerpunkte liegen in der Seelsorge und Religionsgerontologie. 